Arqueología y Sociedad,

$N^{o} 15,2004$

\title{
La arqueometalurgia en el Perú
}

\author{
Luisa Vetter Parodi* \\ Pilar Portocarrero Gallardo**
}

Resumen Este artículo intenta dar una visión de la arqueometría en el estudio de la metalurgia precolombina en el Perú, basado en la utilización de equipos que se encuentran en nuestro país y que pueden ser de utilidad para el investigador, sea arqueólogo o conservador, para el estudio de las piezas de metal antiguas.

Palabras Clave: Arqueometría, Arqueometalurgia, "tumbaga”, bronces, análisis químicos, análisis metalográficos, análisis de microdureza, área Andina. The purpose of this article is to give a vision of the studies about archeometry at
precolumbian metalurgie in Peru, and the use of equipments already existant in our
country that can be useful for researchers to study ancient metals.
Archeometry, Archeometallurgy, "Tumbaga" (alloy of Au-Ag-Cu, Au-Cu, Ag-Cu),
Bronze, Chemical Analysis, Metallographic Analysis, Microhardness Analisis,
Andean Area.

*Diplomatura en Arqueometría, correo electrónico: luchivetter@hotmail.com

** Diplomatura en Arqueometría, correo electrónico: pilarpg@yahoo.com 


\section{Introducción}

La arqueología es una "ciencia" que se ocupa del estudio del hombre a través de los restos materiales dejados por éste. Desde esta definición podemos mencionar a los metales como parte de los restos materiales que alguna vez fueron utilizados por el hombre.

Los metales dentro de la arqueología peruana no han sido bien estudiados hasta ahora, los arqueólogos tienden más a preocuparse por los restos cerámicos, textiles o por los óseos. Esto puede parecer extraño, ya que el Perú es considerado dentro del área Andina como un sitio con un alto desarrollo de la metalurgia en épocas precolombinas, la cual continuó en la Colonia y República, incluso en nuestros días se siguen utilizando técnicas antiguas en la elaboración de piezas de metal. Esto lo demuestran las bellas piezas obtenidas de tumbas o incluso procedentes de huaquería, colecciones que llenan vitrinas de algunos museos de nuestro país y del exterior. A pesar de esta cantidad y variedad de piezas de metal, no han llamado la atención a los investigadores para que sean estudiadas científicamente, sino simplemente se tienen como meras piezas de arte con el único propósito de deleitar al curioso sin aportarle nada más que una satisfacción visual.

Hasta la década de los ochenta las piezas de metal eran estudiadas en base a su iconografía $u$ orfebrería, pero a mediados de esta década se empezó a desarrollar el interés por el estudio arqueométrico. En esta época es que el investigador japonés Izumi Shimada comienza un estudio a largo plazo con respecto a la metalurgia antigua de la costa norte del Perú. Shimada realiza excavaciones en Cerro de los Cementerios, Batán Grande en Lambayeque donde descubre hornos de fundición usados para la aleación de bronce arsenical. Estos hornos tenían 600 años de actividad ininterrumpida. Shimada y su equipo recrean el proceso de fundición usando los hornos antiguos, así como los minerales de cobre y arsénico localizados en la mina de Cerro Mellizos, próxima a los talleres de fundición. El estudio de la arqueometalurgia empieza a dar un giro con estos descubrimientos, tanto Shimada como otros investigadores dan una importancia mayor a las piezas de metal, desde el punto de vista de todo el proceso de fabricación. Es así que en el siglo XXI se han construido museos como el de Sicán en Ferreñafe, Tumbas Reales de Sipán en Lambayeque y Kuntur Wasi en Cajamarca, con piezas provenientes únicamente de excavaciones controladas donde los objetos han sido analizados debidamente, dando como resultado información valiosa desde el punto de vista de la arqueometría.

En el Perú se tiene noticia que se hacen análisis de piezas de metal desde el año 1964. George Marshall, en ese año, realizó análisis metalográficos a unas piezas del M.N.A.A.H.P., exámenes que fueron realizados en la Universidad Nacional de Ingeniería. Desgraciadamente, esto no continuó dándose en el Perú, más bien los investigadores, en su mayoría extranjeros, tomaban muestras de las piezas de metal y se las llevaban a los laboratorios de sus países para que sean analizadas; permitiendo que se forme un vacío en la información y experiencia que se pueda obtener de dicho análisis. Este vacío en la arqueometría se debió en gran parte al desinterés de los profesionales peruanos (físicos, químicos, metalurgistas) en realizar estudios de este tipo, lo que se dio en gran medida por su falta de preparación y conocimientos en la manipulación y tipo de resultados que podría proporcionar las piezas arqueológicas.

Recién hace 12 años se viene dando un interés en el estudio de la arqueometría relacionada con la arqueometalurgia. Ésta es una disciplina joven en nuestro medio, aún hay pocos seguidores de esta disciplina, además todavía no se conocen los equipos que existen en el Perú y que pueden ser usados para analizar metales. También la falta de comunicación, que aún se da,

\footnotetext{
${ }^{1}$ Museo Nacional de Arqueología, Antropología e Historia del Perú.
} 
entre la gente de ciencias y los arqueólogos impide un flujo continuo de información y por lo tanto de resultados.

En el caso de la arqueometalurgia, aún quedan por conocer los alcances de muchos equipos y las ventajas de éstos para el análisis de las piezas de metal. Es por eso que en este trabajo daremos algunos de los equipos que pueden ser usados para esta especialidad y los alcances de cada uno de ellos.

\section{Decisiones para elegir un equipo}

El investigador (arqueólogo o conservador) debe plantearse primero la pregunta de la información que quiere obtener de la pieza que tiene en sus manos. Es muy común llegar a un laboratorio y no tener idea de qué preguntas hacer con respecto a la pieza que desea analizar, ni qué procedimientos seguir para realizar los análisis. Como primera aproximación al estudio de una pieza de metal o sus derivados es necesario elaborar una ficha técnica de dicho objeto con la mayor información que se pueda proporcionar mediante un análisis macroscópico. Aquí se deberá incluir los datos de procedencia (excavación, colección, etc.), foto o dibujo, dimensiones, peso, función (posible) del objeto, proceso de elaboración, técnicas o tratamiento de superficie, técnicas decorativas, estado de conservación y si ha recibido algún tratamiento previo.

Con esta información, el investigador tendrá una idea más clara del tipo de pieza que tiene entre sus manos y podrá hacerse las preguntas necesarias para entender mejor el proceso que se le siguió al objeto en su elaboración. Con estas preguntas el investigador recién podrá ir al laboratorio para planteárselas al técnico y decidir entonces qué tipo de análisis realizar. Esto no termina en esta etapa, ya que el investigador deberá saber cómo se tratará su pieza, si es sario una muestra de ésta y cuánto, si esta muestra será destruida o podrá ser reutilizada para otro tipo de análisis; o quizás se usará toda la pieza sin destruirla, si es así en qué condiciones se la devolverán y cómo afectará ese procedimiento a la pieza en el futuro. Otra pregunta de rigor es cuánto tiempo se demorará el examen y el valor de éste.

\section{Los análisis y equipos}

Los equipos que a continuación se describen pertenecen a distintos laboratorios del país. Estos equipos han sido usados para la realización de análisis a piezas de metal precolombina, colonial y republicana. Además, también han sido útiles para el proceso de conservación de este tipo de piezas, ya que permiten tener conocimiento del tipo y grado de corrosión que tiene la pieza a conservar. Si bien no son todos los equipos que en la actualidad están a disposición de los investigadores interesados en analizar piezas de metal, ${ }^{2}$ es una gran aproximación al conocimiento de su existencia, uso y cualidades de cada uno de ellos.

\section{Análisis químicos:}

- Permiten establecer la composición de la muestra.

- Cuantitativo: Cuánto.

- Cualitativo: Qué o cuál.

- Semi cuantitativo: Indica la mayoría de elementos presentes en la muestra y da porcentajes aproximados del contenido de cada uno de ellos.

$\begin{array}{cc}\text { Elementos: } & \text { Mayores } \\ >10 \% & \begin{array}{l}\text { Menores } \\ 10 \%-1 \%\end{array} \\ & \\ \text { Trazas } & \text { Vestigios } \\ 1 \%-.001 \% & <.001 \%\end{array}$

\footnotetext{
${ }^{2}$ Los equipos que son descritos en este trabajo corresponden a los utilizados por las autoras en sus estudios arqueometalúrgicos. Si el lector desea conocer más sobre equipos usados para la arqueometría puede consultar: Estrada, Walter. Informe de Consultoría. Capacidades de investigación científica y tecnológica en el área temática de Ciencia de los Materiales. 45 Informe de Consultorías presentados al BID como parte del Proyecto: Programa Nacional de Ciencia y Tecnología. CONCYTEC. 2003.
} 


\section{Equipos:}

\section{Resultados semi cuantitativos:}

- Espectrografía de emisión óptica (UNI)³: Permite análisis semi cuantitativos. La muestra, en trozos muy pequeños o pulverizada, es excitada y vaporizada por medio de una descarga eléctrica llegando a temperaturas de aprox. $5000^{\circ} \mathrm{C}$, emite radiación visible, que pasa por un sistema óptico antes de imprimir una película fotográfica que se analiza en un microdensitómetro.

Ventajas y desventajas. - Es un tipo de análisis químico que proporciona información semi cuantitativa de la composición elemental de la pieza en estudio, por lo que es uno de los primeros análisis que deben realizarse. Teniendo una idea de la composición elemental de la muestra, el investigador podrá decidir sobre qué elementos es necesario realizar análisis cuantitativos. Entre las ventajas de este método está que pese a que nos brinda simultáneamente información de muchos elementos químicos el costo no es muy alto. La desventaja es que es un método destructivo y que los resultados son aproximados, con errores relativamente grandes. Se necesitan aprox. $25 \mathrm{mg}$. de muestra.

Fluorescencia de Rayos $X$ (IPEN) ${ }^{4}$ : La muestra se irradia con radiación gama o rayos $\mathrm{X}$ producidos por una fuente externa. Los fotones de la radiación utilizada deben tener energía suficiente para provocar la expulsión de electrones localizados en capas interiores en los átomos presentes en la matriz. Los electrones de capas exteriores emiten radiaciones características durante el proceso de fluorescencia que conlleva a la ocupación de las vacancias producidas en las capas interiores.

Ventajas y desventajas. - Es un tipo de análisis químico que proporciona información semi cuantitativa de la composición elemental de la pieza en estudio, brindando información de muchos elementos químicos. Además es método no destructivo.

- Microscopio electrónico de barrido con microsonda electrónica (PUCP - UNMSM): Combina el microscopio electrónico de barrido con un analizador de rayos X. Se pueden efectuar exámenes morfológicos microscópicos y simultáneamente determinaciones composicionales. Es un tipo de análisis no destructivo dependiendo del tamaño de la pieza.

- Difracción de Rayos X (UNMSM) ${ }^{5}$ : Su uso se basa en la interferencia entre haces de radiación electromagnética monocromática, reflejados por la red cristalina formada por los átomos que constituyen el sólido. Se usa para identificar compuestos, siendo muy útil para analizar minerales, pigmentos o productos de corrosión.

Ventajas y Desventajas. - Es un tipo de análisis que no proporciona información de la composición elemental del material en estudio, pero brinda información de la estructura del compuesto y puede distinguir entre fases diferentes del mismo compuesto químico. Es un método destructivo porque la muestra debe ser pulverizada, aunque el polvo resultante puede utilizarse para otros ensayos. Requiere de 100 a $250 \mathrm{mg}$ de muestra.

\section{Resultados cuantitativos:}

- Absorción Atómica (PUCP, UNI, UPCH'): Se basa en la absorción resonante de la radiación emitida desde una lámpara por las especies químicas presentes en la muestra, que debe ser nebulizada y atomizada antes de ser expuesta a la luz de la fuente, cuya intensidad se mide luego de atravesar la muestra. La cantidad de luz absorbida por la muestra se relaciona al contenido del elemento químico que se analiza.

\footnotetext{
${ }^{3}$ Universidad Nacional de Ingeniería.

${ }^{4}$ Instituto Peruano de Energía Nuclear.

${ }^{5}$ Universidad Nacional Mayor de San Marcos.

${ }^{6}$ Universidad Peruana Cayetano Heredia.
} 
Ventajas y Desventajas. - Es un método rápido y no muy costoso para pocos elementos. Los resultados son bastante precisos. Entre las desventajas que presenta está que se necesitan aproximadamente $25 \mathrm{mg}$ de muestra, que se disuelven y por lo tanto no pueden volver a utilizarse para otros ensayos lo que lo convierte en un método absolutamente destructivo. El equipo requiere de lámparas especiales para cada elemento por lo que es costoso si se quiere hacer medidas de varios elementos.

Activación Neutrónica (IPEN): Es un tipo de análisis multielemental cualitativo y cuantitativo, basado en la inducción de radiactividad artificial en la muestra mediante el bombardeo de ésta con neutrones. La identificación de los elementos se realiza posteriormente por sus emisiones de radiación gama.

Ventajas y desventajas. - Es una técnica sumamente poderosa que proporciona datos precisos en un amplio rango de concentraciones, desde ppm hasta porcentaje. Identifica y cuantifica la mayoría de los elementos químicos. Se necesitan aproximadamente $10 \mathrm{mg}$ de muestra. Dependiendo del tamaño de la muestra que se analiza podría ser no destructivo, y puede servir para análisis posteriores por el mismo método $\mathrm{u}$ otro. La mayor desventaja está en la preparación de las muestras y en la espera de algunos días o meses luego de la irradiación antes de manipularla con seguridad. Debido a que requiere el uso de un reactor nuclear u otra fuente de neutrones es un método costoso. Los investigadores deben acudir a este tipo de análisis cuando se requieran de resultados sumamente precisos.

Espectroscopia Mössbauer (UNMSM): Se basa en la emisión y absorción resonante de radiación gama por los núcleos de ciertos isótopos, sin que se produzca retroceso. Los núcleos en los que se puede producir el efecto Mössbauer no son muchos, pero afortunadamente el Fe-57 es uno de ellos, por lo que esta técnica es una de las más importantes para la identificación de compuestos de hierro, principalmente los óxidos. Esta información puede ser usada para determinar el tipo de atmósfera (oxidante o reductora) y la temperatura máxima alcanzada en los hornos de cocción de cerámicos o de fundición de metales. Si la medida se realiza por transmisión la muestra debe ser pulverizada y por lo tanto es un método destructivo, aunque la muestra puede ser utilizada luego para otros ensayos. Si la muestra contiene gran cantidad de hierro y no se la quiere pulverizar, entonces la medida se puede realizar por reflexión en la superficie y de esta manera el fragmento no se destruye. Se necesita $250 \mathrm{mg}$. de muestra.

\section{Resultados sobre procesos de manufactura:}

Estudios metalográficos: Permiten describir la microestructura dando información sobre el origen y autenticidad de la pieza, el proceso de elaboración, los tipos de tratamiento superficial (dorado o plateado), los tratamientos térmicos de recocido y templado, la naturaleza del metal o aleación y los productos de corrosión, así como del estado de conservación.

Se puede aplicar utilizando un microscopio electrónico de barrido con analizador de espectrómetro dispersivo de energía (PUCP, UNMSM) o un microscopio metalográfico. Es una técnica no destructiva dependiendo del tamaño de la muestra y del tipo de microscopio que se emplee.

Análisis de microdureza (PUCP, UNI, UNMSM) Caracteriza el metal por su índice de resistencia y tenacidad. Requiere contar con patrones de aleación y manufacturas similares. Es no destructiva dependiendo del tamaño de la muestra.

Análisis radiográfico (PUCP, UNI, UNMSM) ayuda al estudio de la estructura interna de la pieza, se puede observar soldaduras, fracturas, corrosión interna, etc. No es destructivo.

\section{Interpretación de los resultados}

Los resultados no necesariamente responderán todas las preguntas, a veces crean otras. En este caso es recomendable analizarlos con un especialista en el área de la química o metalurgia 
o geología, según sea el caso, para interpretarlos como es debido. Si el objeto procede de una excavación controlada, entonces los resultados serán complementarios a la información de la excavación. Pero si su procedencia es desconocida, como sucede con muchas piezas que se encuentran en los museos y colecciones privadas, la información de los análisis será la única que nos permita reconstruir de la mejor forma posible el pasado de dicho objeto.

En cambio, los análisis para determinar el tipo de corrosión que tiene la pieza para proceder a su posterior conservación, serán quizás más fáciles de interpretar, ya que el especialista que lea estos resultados debe ser experto en química, capacitado para realizar el trabajo de conservación. Los resultados le ayudarán a decidir qué tipo de químicos debe usar para la limpieza de la pieza y su posterior conservación en un ambiente idóneo para el tipo de aleación con la que esté fabricada.

Como se mencionó en la introducción, el interés por la arqueometalurgia en el Perú es muy reducido, lo que ocasiona un vacío en la interpretación de los resultados.

En el Viejo Mundo este tipo de estudios se encuentra muy desarrollado. Tenemos el ejemplo de las investigaciones realizadas en los laboratorios de Arqueología y de Historia del Arte de la Universidad de Oxford, donde en la década de los ochenta se analizó alrededor de 600 piezas (monedas en su mayoría) de latón (cobre-zinc) provenientes de Inglaterra, Nüremburg, Francia y otros países europeos pertenecientes a los años 1280 a 1900 d.C.

El resultado de este estudio le permitió observar a los investigadores que durante el siglo XV hubo un cambio importante en la forma de elaborar las piezas de latón. Antes, el contenido de este tipo de piezas era de $4 \%$ de estaño, 1-2\% de plomo con un $10 \%$ de zinc como máximo. Trazas de antimonio, plata y arsénico están presentes en 0.1-0.2\%; mientras el níquel es muy bajo, usualmente menor al 0.05\%. Después de 1450 d.C. el contenido de zinc llegaba a un $20 \%$, con una caída del plomo (menos del 1\%) y del estaño (menos del 0.2\%). Los elementos traza se encuentran usualmente por debajo del $0.1 \%$, a excepción del níquel que es mayor al $0.5 \%$

Según los investigadores ingleses, este tipo de cambio en la proporción del zinc dentro de la aleación se debió a dos hechos posibles: uno es el cambio en el mineral de origen del cobre proveniente del noreste de Europa (de un mineral rico en Sn, Sby Ag a uno con mayor contenido de Ni). Y la segunda, es el cambio en la técnica de manufactura, de una en la cual trozos de bronce son incluidos normalmente en el proceso de cementación, a una en donde se permitía el uso de trozos de latón o cobre (Pollard et. al. 1996). Este tipo de investigación permite conocer la antigüedad de las piezas de latón, ya que el tipo de trazas presentes en las piezas elaboradas con esta aleación son bastante características para las fechas señaladas líneas arriba; de esta forma se permitirá identificar no sólo a qué período corresponde la pieza analizada, sino también su posible identificación como objeto verdadero ofalso.

$\mathrm{Si}$ bien estos estudios fueron realizados a piezas del siglo XI al XX donde los datos históricos están mejor identificados que en épocas anteriores (periodo sin escritura, por ejemplo) y permiten una relación de los resultados con los hechos históricos conocidos; en el Perú no contamos con este tipo de investigaciones tan amplias donde se permita relacionar adecuadamente un tipo de aleación con hechos históricos o arqueológicos. Este tipo de estudios debe de realizarse para el área Andina, no sólo para periodos prehispánicos, sino también para la Colonia y República.

Heather Lechtman es una investigadora norteamericana del Instituto Tecnológico de Massachussets (MIT) dedicada al estudio de los metales en épocas precolombinas desde los años setenta, y es quien le ha puesto mayor interés al estudio de las aleaciones usadas por los antiguos metalurgistas en el área Andina. No sólo ha trabajado con los bronces sino también con la 
binaria de cobre-oro; o cobre-plata) y sus posibles formas de uso.

El bronce es conocido comúnmente como una aleación de cobre con estaño, pero en el área Andina no sólo existe este tipo de bronce. A diferencia del Viejo Mundo donde se conocen tres tipos de bronces (cobre-estaño, cobrearsénico y cobre-estaño-plomo), en esta parte del continente americano, Lechtman (1978, $1996,1997)$ ha identificado cinco tipos de bronces, estos son: bronces de aleación binaria (cobre-estaño) y (cobre-arsénico), aleación ternaria (cobre-arsénico-níquel), (cobre-arsénicoestaño) y (cobre-arsénico-zinc). Todos estos bronces se desarrollaron en los Andes del Sur ${ }^{7}$, a excepción de las aleaciones de cobre-arsénico y cobre-arsénico-estaño que también se desarrollaron en los Andes Centrales. Todos aparecen en el Horizonte Medio (550-900 d.C.), pero para el Horizonte Tardío con los Incas (1470-1532 d.C.) el bronce que se impondrá será el binario de cobre-estaño. Una característica importante en estos bronces es que tienen la misma propiedad mecánica de dureza.

Según Lechtman $(1996,1997)$, estas aleaciones se usaron por la cercanía de los yacimientos de minerales que las componen. Este punto está demostrado en la costa norte del Perú, donde la aleación que se desarrolló como bronce fue la de cobre con arsénico.

Como se menciona en la parte introductoria, Shimada y su equipo (Merkel et. al. 1994) identificaron las minas que contenían los minerales de cobre (malaquita) y de arsénico (arsenopirita) que fueron usados para este bronce. Las minas se encuentran cerca del área del Santuario Histórico Bosque de Pomac, en Batán Grande, Lambayeque; lugar que fue considerado la capital de la cultura Sicán hasta el año 1100 d.C. Dicha cultura fue la que desarrolló el bronce arsenical alrededor del 900 d.C. y lo produjo a gran escala. Este bronce ha sido estudiado por
Shimada y su equipo (Shimada 1985, 1987, 1990, 1995, Shimada et. al. 1982, 1983, 1991), Lechtman (1976, 1978, 1981, 1996, 1997), una de las autoras (Vetter 1993, 1996, 1997, 2003) y por Bezúr (2003).

Otra aleación interesante pero tardía es la de cobre con zinc, lo que se conoce como latón. Lechtman (1997) ha identificado este tipo de piezas para la época de la Colonia, al igual que una de las autoras (Vetter, en prensa). En ambos casos se tratan de tupus o prendedores elaborados con este tipo de aleación y que corresponden a la época de transición o colonial, es decir, entre los siglos XVI y XVII. Es probable que este tipo de aleación haya sido traída por los españoles, ya que aún no se conocen piezas elaboradas en base al latón para la época precolombina.

Los resultados de los análisis referidos a metales y sus derivados están muy ligados a la composición química elemental (cualitativa y cuantitativa) y a la microestructura de éstos. Normalmente, el investigador busca en una pieza de metal este tipo de respuestas que lo ayudarán a definir el elemento o aleación con la cual fue fabricada y el proceso de manufactura que se le siguió a la pieza. Según estos resultados es que investigadores como Lechtman han podido identificar las cinco variedades de bronce que se han expuesto líneas arriba. Del mismo modo se ha podido determinar algunas de las similitudes y diferencias en las propiedades mecánicas de los bronces arsenicales y estañíferos. Esta interpretación proporcionó datos que ayudaron a entender que el metalurgista antiguo buscó estas aleaciones según los minerales que tenía a la mano, pero que le proporcionase la dureza (propiedad mecánica de mayor importancia y que coincide con todos los bronces) necesaria para el tipo de pieza que se fabricaría (en muchos casos fueron piezas para uso agrícola o de construcción civil). También fue importante la identificación del uso del color en las aleacio-

\footnotetext{
${ }^{7}$ Los Andes del Sur comprenden los territorios del altiplano Perú-Bolivia, norte de Chile y noroeste de Argentina.
} 
nes. En el caso de los bronces, a mayor cantidad de arsénico el metal se vuelve de color plateado y a mayor cantidad de estaño se torna dorado. Los porcentajes altos, tanto de arsénico como de estaño, en la aleación hacen perder la propiedad mecánica de dureza, pero le crea una nueva propiedad de color que fue muy difundida en Ecuador y México después de haber sido usada en Perú (Hosler, 1994).

Otro ejemplo de esta propiedad de color es la que se encuentra en las aleaciones tumbaga, muy conocida desde la cultura Vicús (200-550 d.C.), aunque sus orígenes podrían estar en el Horizonte Temprano (1000-400 a.C.); con la pieza de tumbaga (cobre-plata) hallada por Patterson en 1966 en el sitio de Malpaso, en Lurín (Lechtman, 1978). Esta pieza tenía un proceso de enriquecimiento en la superficie, lo que le dio color plata.

Ha habido casos en que se pensaba que la pieza era de oro o de plata, y en realidad sólo tenían un porcentaje muy bajo de estos elementos (alrededor del 10\%), siendo el cobre el elemento de mayor porcentaje. Lechtman (1984) ha estudiado este tipo de aleaciones y sus variados tratamientos para lograr una superficie de "oro" o de "plata".

Una de las culturas que desarrolló muy bien esa técnica fue la cultura Mochica (200-750 d.C.). Un ejemplo conocido es el ajuar del Señor de Sipán (ca. 350 d.C.), donde parte de las piezas de metal corresponden a este tipo de aleación cuya superficie fue tratada para dar el aspecto de plata u oro. Son limitados los contextos intactos donde se han hallado piezas de tumbaga, es por esto que en este contexto del Señor de Sipán es muy importante el estudio de tales piezas, ya que se puede relacionar su ubicación dentro de la tumba pudiendo entender de esta manera la simbología e ideología que este tipo de piezas representaban como parte del ajuar funerario del individuo.

Para poder identificar el tipo de tratamiento que se le dio a estas piezas, es necesario usar un microscopio electrónico que ayude a identificar las diferentes fases que se encuentran en el objeto y cómo han sido éstas tratadas. De esta forma se podrá saber si la superficie corresponde a un enchapado, a un enriquecimiento por eliminación del cobre por medio de ácidos corrosivos o a un dorado o plateado por fusión o reemplazo electroquímico.

\section{Reflexiones finales}

Este trabajo lo único que intenta es dar a conocer la tecnología con la que contamos en el Perú. Sabemos que no es de última generación en algunos casos, pero son equipos que sirven para el estudio de las piezas de metal y sus derivados, y cuyos resultados son de garantía para el investigador.

Es conveniente incentivar a los arqueólogos en general y a los químicos, físicos y metalurgistas en particular para que aporten con sus conocimientos al estudio de la arqueometalurgia dentro de la arqueometría. Es necesario hacer entender a las nuevas generaciones de arqueólogos que la arqueometría, y por ende la arqueometalurgia, es parte de la arqueología y por lo tanto aporta datos muy importantes para tener un entendimiento global de la zona de estudio (siempre y cuando haya evidencia metalúrgica en el sitio de investigación).

Si bien nos hemos centrado en la etapa precolombina de la historia del Perú, es necesario poner atención en la época de la Colonia y República. Aunque el estudio de la minería en estas dos etapas de la historia del Perú ha sido muy desarrollado, ésta no ha sido aún relacionada con la orfebrería. Esta falta de vinculación se debe lamentablemente a que muchas de las piezas de esas épocas han sido refundidas con el fin de usar el metal para elaborar nuevas piezas con diseños más modernos.

Por último, es urgente elaborar un patrón de aleaciones de metales y formas de manufactura para el área Andina, de tal modo que nos ayude a identificar de mejor manera las piezas arqueológicas e históricas y sus posibles relaciones con las áreas vecinas. 


\section{Anexos}

En el Anexo I se ha elaborado una "Historia de los análisis en la metalurgia de piezas peruanas" acompañada de la bibliografía de donde se ha obtenido esta información. Cabe resaltar que quizás no sea toda la bibliografía existente en el tema, pero se ha hecho el esfuerzo de tratar de hacerla lo más completa posible.

En el Anexo II se colocan los equipos que pueden ser usados para analizar muestras de cada una de las etapas de la metalurgia: minería, metalurgia y orfebrería. Basado en la investigaciones del Proyecto Arqueológico Sicán.

\section{Agradecimiento}

Deseamos agradecer a Paloma Carcedo, Susana Petrick y Alejandro Trujillo por sus importantes comentarios a este trabajo.

\section{Bibliografía}

Bezúr, Aniko

2003 "Variability in Sicán copper alloy artifacts: its relation to material flow patterns during the middle Sicán period in Peru, A.D. 900-1100". A dissertation submitted to the Faculty of the Department of Materials Science and Engineering in partial fulfillment of the requirements for the Degree of Doctor of Philosophy in the Graduate College The University of Arizona.

Britton D. Y Eva Richards

1980 "La espectroscopia de emisión óptica y el estudio de la metalurgia durante la Edad del Bronce europea", en Brothwell y Higgs (Comp.). Ciencia en Arqueología. México: Fondo de Cultura Económica, pp. 631-642.

Hosler, Dorothy

1994 The Sounds and Colors of power: The Sacred Metallugical Technology of Ancient West Mexico. MIT Press. Cambridge.

Lechtman, Heather

1976 "A metallurgical site survey in the
Peruvians Andes". Journal of Field Archaeology, 3: 1-42. Boston.

1978 “Temas de Metalurgia Andina”, en Ravines (comp.). Tecnología Andina. Lima: IEP.

1981 "Copper-arsenic bronzes from the north coast of Peru. The Research Potential of Anthropoligical Museums Collections". Annals of the New York Academy of Sciences, 376: 77-122. New York.

1984 "Pre-Columbian surface metallurgy". Scientific American, 250 (6): 56-63.

1988 Traditions and styles in the Central Andes metalworking. The beginning of the use of metals and alloys: 344-378. Ed. R. Maddin. MIT Press. Cambridge.

1996 "El bronce y el Horizonte Medio". Boletín Museo del Oro. 41: 3-25. Bogotá: Banco de la República.

1997 "El Bronce arsenical y el Horizonte Medio", en R. Varón G. y J. Flores E. (ed.). Arqueología, Antropología e Historia en los Andes: Homenaje a María Rostworowski: 153-186. Lima: IEP.

Merkel J., I. Shimada, C. Swann y R. Doonan

1994 "Investigation of the prehistoric copper production at Batán Grande, Perú: interpretation of the analytical data for ore sample". Archaeometry of PreColumbian sites and artifacts: 199-227. Eds. D.A. Scout y P. Meyers. The Getty Conservation Institute. Marina del Rey. CA.

Pollard, M.y C. Heron

1996 Archaeological chemistry. The Royal Society of Chemistry. 392 p. London.

Shimada, Izumi

1985 "La Cultura Sicán: Caracterización Arqueológica”, en E. Mendoza (comp.). Presencia Histórica de Lambayeque: 76133. Lima: DESA S. A.

1987 "Aspectos tecnológicos y productivos de la metalurgia Sicán, costa norte del Perú”. Gaceta Arqueológica Andina, año IV, 13: 15-21. Lima.

1990 Cultural Continuities and 
Discontinuties on the Northern North Coast of Peru, Middle-Late Horizons. The Northern Dynasties: Kingship and Statecraft in Chimor: 297-392 Moseley, Michael E. \& Alana Cordy-Collins, eds. Dumbarton Oaks. Washington, D.C.

1995 Cultura Sicán. Dios, riqueza y poder en la costa norte del Perú. 219 p. Lima: Fundación del Banco Continental para el Fomento de la Educación y la Cultura, EDUBANCO.

Shimada, Izumi; Stephen Epstein y Alan Craig

1982 "Batán Grande: a prehistoric metallurgical center in Peru”. Science, 216: 952 959.

1983 "The metallurgical process in ancient north Peru". Archaeology, 36, (5): 38-45.

Shimada Izumi y John Merkel

1991 "Copper-Alloy Metallurgy in Ancient Peru”. Scientific American, vol 265, NŸ 1:80-86.

Vetter, Luisa

1993 "Análisis de las Puntas de Aleación de Cobre de la Tumba de un Señor de la Elite Sicán, Batán Grande, Lambayeque, Perú". Tesis de Bachiller.

Pontificia Universidad Católica del Perú, setiembre.

1996 "El uso del Cobre Arsenical en las Culturas Prehispánicas del Norte del Perú”. 49 Congreso Internacional de
Americanistas, Quito, 1997. Boletín Museo del Oro, NŸ41, Julio-diciembre, pp. 63-81. Bogotá.

2004 "Las Piezas de Metal del Museo de Sitio Arturo Jiménez Borja Puruchuco". En: Puruchuco y la sociedad de Lima: un homenaje a Arturo Jiménez Borja. Editores: L. F. Villacorta, L. Vetter y C. Ausejo.

(En prensa)La evolución del Tupu en forma y manufactura desde los Incas hasta el Siglo XIX. 51 Congreso Internacional de Americanistas, Santiago de Chile, Chile, julio, 2003.

Vetter, L., P. Carcedo, S. Cutipa y E. Montoya

1997 "Estudio Descriptivo, Metalográfico y Químico de las Puntas de Aleación de Cobre de la Tumba de un Señor de la Elite Sicán, Lambayeque, Perú, empleando Técnicas de Microscopía Óptica y Análisis por Activación Neutrónica”. Revista Española de Antropología Americana, 27, 23-38. Servicio Publicaciones UCM. Madrid.

Vetter, L. y Jesús Ruiz

2003 "Técnicas analíticas en las piezas de metal arqueológicas". Segundo Congreso Internacional de Metalurgia de Transformación, Cuzco, 12 al 14 de noviembre. CD-Rom. 


\section{ANEXO I}

\section{Historia de los análisis en la metalurgia de piezas peruanas}

\begin{tabular}{|c|c|c|}
\hline Baessler, 1906 & Análisis químicos cuantitativos & Costa central del Perú \\
\hline Foote y Buell, 1912 & Análisis químicos cuantitativos & Bronces del Perú \\
\hline Mathewson, 1915 & $\begin{array}{l}\text { Análisis metalográficos } \\
\text { Análisis químicos cuantitativos }\end{array}$ & Cu-Sn de Machu Picchu, sierra sur \\
\hline Nordenskiold, 1921 & Análisis químicos cuantitativos & Perú \\
\hline Lothrop, 1953 & Análisis químicos cuantitativos & Piezas de oro y plata del Perú \\
\hline Hollister, 1955 & Análisis químicos cuantitativos & Escorias de Chan Chan, costa norte \\
\hline Caley y Easby, 1959 & Análisis químicos cuantitativos & Lingote de Ica, costa sur \\
\hline Marshall, 1964 & Análisis metalográficos & $\begin{array}{l}\text { Piezas del Museo Nacional de } \\
\text { Arqueología, Antropología e Historia } \\
\text { del Perú, análisis realizados en la } \\
\text { Universidad Nacional de Ingeniería, } \\
\text { Perú }\end{array}$ \\
\hline Root, 1965 & $\begin{array}{l}\text { Análisis metalográficos } \\
\text { Análisis químicos cuantitativos }\end{array}$ & Costa sur del Perú \\
\hline Caley, 1970 & Análisis químicos cuantitativos & Objetos de cobre del Perú \\
\hline Petersen, 1970 & $\begin{array}{l}\text { Análisis químicos cuantitativos } \\
\text { Análisis espectrográficos }\end{array}$ & $\begin{array}{l}\text { Oro y cobre nativos del Perú; vaso de } \\
\text { plomo de Batán Grande, costa norte }\end{array}$ \\
\hline Friedman et al., 1972 & Análisis químicos cuantitativos & Copa Moche, costa norte \\
\hline Ríos y Retamozo, 1978 & $\begin{array}{l}\text { Análisis espectroquímicos } \\
\text { cualitativos }\end{array}$ & $\begin{array}{l}\text { Piezas de plata de la Isla San Lorenzo, } \\
\text { costa central }\end{array}$ \\
\hline Gordon y Rutledge, 1987 & $\begin{array}{l}\text { Análisis metalográficos } \\
\text { Microscopia electrónica de } \\
\text { barrido (SEM) }\end{array}$ & $\begin{array}{l}\text { Piezas de bronce de Machu Picchu, } \\
\text { sierra sur }\end{array}$ \\
\hline Rovira, 1991 & Análisis metalográficos & Perú \\
\hline Diez Canseco, 1994 & $\begin{array}{l}\text { Análisis metalográficos } \\
\text { Análisis químicos cuantitativos }\end{array}$ & $\begin{array}{l}\text { Piezas Vicús, costa norte. Análisis } \\
\text { realizados en el Perú }\end{array}$ \\
\hline Bezúr, 1996, 2003 & $\begin{array}{l}\text { Análisis químicos por: } \\
\text { Fluorescencia de rayos X (FRX) } \\
\text { Emisión atómica inducida por } \\
\text { plasma en espectroscopia de } \\
\text { emisión óptica (ICP-OES) } \\
\text { Microscopio electrónico de } \\
\text { barrido(SEM)con espectroscopia }\end{array}$ & $\begin{array}{l}\text { Cu-As del valle del Mantaro en la } \\
\text { sierra sur-central y de Batán Grande, } \\
\text { costa norte }\end{array}$ \\
\hline
\end{tabular}




\begin{tabular}{|c|c|c|}
\hline & $\begin{array}{l}\text { de energía dispersa (EDS) } \\
\text { Espectrómetro de longitud de } \\
\text { onda dispersa (WDS) }\end{array}$ & \\
\hline Centeno et al., 1996 & $\begin{array}{l}\text { Microscopia electrónica de } \\
\text { barrido (SEM) }\end{array}$ & $\begin{array}{l}\text { Piezas de cobre dorado de Loma } \\
\text { Negra, costa norte }\end{array}$ \\
\hline Makowski et al., 1996 & $\begin{array}{l}\text { Análisis metalográficos } \\
\text { Microscopia electrónica de } \\
\text { barrido (SEM) }\end{array}$ & $\begin{array}{l}\text { Piezas Vicús, costa norte. Análisis } \\
\text { realizados en el Perú }\end{array}$ \\
\hline Schorsch et al., 1996 & $\begin{array}{l}\text { Microscopio electrónico de } \\
\text { b a r rido ( S E M ) co n } \\
\text { espectroscopia de energía } \\
\text { dispersa (EDS) } \\
\text { Activación Neutrónica }\end{array}$ & $\begin{array}{l}\text { Piezas de cobre dorado de Loma } \\
\text { Negra, costa norte }\end{array}$ \\
\hline Mc Ewan et al., 2000 & Análisis químicos cuantitativos & Costa sur del Perú \\
\hline Scott, 2000 & $\begin{array}{l}\text { Fluorescencia de rayos X (FRX) } \\
\text { Microscopio electrónico de } \\
\text { barrido con espectroscopia de } \\
\text { energía dispersa (EDS) }\end{array}$ & Costa norte del Perú \\
\hline Castro de la Mata, 2003 & $\begin{array}{l}\text { Análisis metalográficos } \\
\text { Microscopio electrónico de } \\
\text { b a r r d o ( S E M ) c o n } \\
\text { espectroscopia de energía } \\
\text { dispersa (EDS) }\end{array}$ & $\begin{array}{l}\text { Piezas de cobre dorado de Tablada de } \\
\text { Lurín, costa central. Análisis realiza- } \\
\text { dos en el Perú }\end{array}$ \\
\hline $\begin{array}{l}\text { Lechtman del } 70 \text { al } \\
\text { presente }\end{array}$ & $\begin{array}{l}\text { Análisis metalográficos } \\
\text { Análisis de microdureza } \\
\text { Radiografías } \\
\text { Análisis químicos por: } \\
\text { Vía húmeda } \\
\text { Gravimetría } \\
\text { Análisis isotópicos } \\
\text { Fluorescencia de rayos X (FRX) } \\
\text { Microscopio electrónico de } \\
\text { b a r r i d o ( S E M ) c o n } \\
\text { espectroscopia de energía } \\
\text { dispersa (EDS) } \\
\text { Análisis espectrográficos } \\
\text { Emisión atómica inducida por } \\
\text { plasma en espectroscopia de } \\
\text { emisión (ICP-ES) } \\
\text { Emisión atómica inducida por }\end{array}$ & Costa y sierra del Perú \\
\hline
\end{tabular}




\begin{tabular}{|c|c|c|}
\hline & $\begin{array}{l}\text { plasma en espectroscopia de } \\
\text { masa (ICP-MS) } \\
\text { Absorción Atómica } \\
\text { Microsonda de electrones } \\
\text { Activación Neutrónica } \\
\text { Graduación yodométrica } \\
\end{array}$ & \\
\hline $\begin{array}{l}\text { Shimada del } 80 \text { al } \\
\text { presente }\end{array}$ & $\begin{array}{l}\text { Análisis metalográficos } \\
\text { Análisis químicos por: } \\
\text { Emisión de rayos X inducida por } \\
\text { protones (PIXE) } \\
\text { Difracción de rayos X (DRX) } \\
\text { Microscopio electrónico de } \\
\text { b a r r i d o ( S E M ) c o n } \\
\text { espectroscopia de energía } \\
\text { dispersa (EDS) } \\
\text { Mossbauer } \\
\text { Activación Neutrónica } \\
\text { Absorción atómica }\end{array}$ & $\begin{array}{l}\text { Batán Grande, costa norte (incluye } \\
\text { hornos) }\end{array}$ \\
\hline Vetter del 90 al presente & $\begin{array}{l}\text { Análisis metalográficos } \\
\text { Análisis de microdureza } \\
\text { Radiografías } \\
\text { Análisis químicos por: } \\
\text { Volumetría } \\
\text { Fluorescencia de rayos X (FRX) } \\
\text { Microscopio electrónico de } \\
\text { b a r r i d o ( S E M ) c o n } \\
\text { espectroscopia de energía } \\
\text { dispersa (EDS) } \\
\text { Activación Neutrónica } \\
\text { Absorción atómica } \\
\text { Espectrógrafo de Emisión Óptica }\end{array}$ & $\begin{array}{l}\text { Costa y sierra del Perú } \\
\text { Análisis realizados en distintos labo- } \\
\text { ratorios del Perú (IPEN, UNI, PUCP, } \\
\text { SGS) }\end{array}$ \\
\hline
\end{tabular}




\section{Bibliografía del Anexo I}

Baessler, Arthur

1906 Altperuanische Metallgerate. Verlag von Georg Reimer, Berlín.

Bezúr, Aniko

1996 "Abandoning Arsenic? Technological and Cultural Changes in the Mantaro Valley, Peru". 49 Congreso Internacional de Americanistas, Quito, 1997. Boletín Museo del Oro. NŸ41, pp. 119-130. julio-diciembre, Bogotá.

2003 "Variability in Sicán copper alloy artifacts: its relation to material flow patterns during the middle Sicán period in Peru, A.D. 900-1100". A dissertation submitted to the Faculty of the Department of Materials Science and Engineering in partial fulfillment of the requirements for the Degree of Doctor of Philosophy in the Graduate College The University of Arizona.

Caley, E. R.

1970 Chemical composition of the ancient copper objects of South America. Paper read in June 1970 at a meeting held at the Museum of Fine Arts in Boston. Manuscript.

Caley, E. R. y D. Jr. EASBY

1959 "The Smelting of Sulfide Ores of Copper in Preconquest Peru". American Antiquity, vol. 25, Nü, pp. 59-65.

Cáceres, P.; P. Carcedo; L. Vetter; H. Tantaleán;

S. Cutipa; F. Calderón y J. Ruíz

1999 "Uso del cobre y sus aleaciones durante el incanato en la zona de Cutimbo, Puno". Trabajo presentado en el VI Encuentro de Historiadores de la Minería Latinoamericana, en la Pontificia Universidad Católica del Perú, agosto.

Carcedo, P. y L. Vetter

2002 "Instrumentos utilizados para la fabricación de piezas de metal para el período inca". 50 Congreso Internacional de
Americanistas, Varsovia, Polonia. Julio, 2000. Baessler Archiv. NŸ 50. pp. 47-66. Ethnologisches Museum de Berlín, Alemania.

Carcedo, Paloma, Luisa Vetter y Magdalena Diez Canseco

(en prensa) "Los vasos efigie antropomorfos: un ejemplo de la orfebrería de la costa central durante el Intermedio TardíoHorizonte Tardío". Trabajo presentado en el IV Simposio Internacional de Arqueología PUCP "Identidad y transformación en el Tawantinsuyu y en los Andes coloniales. Perspectivas Arqueológicas y Etnohistóricas”. 16 al 18 agosto del 2002. Boletín de Arqueología, PUCP.

Castro de la Mata, Pamela

2003 "Los artefactos de metal y el ritual funerario en el cementerio de entierros en pozo de Tablada de Lurín". Ponencia presentada en el 51 ICA, Santiago de Chile.

Centeno, S. y D. Schorch

1996 "Caracterización de depósitos de oro y plata sobre artefactos de cobre del valle del Piura (Perú) en el periodo intermedio”. 49 Congreso Internacional de Americanistas, Quito, 1997. Boletín Museo del Oro. NŸ41, pp. 165-186. juliodiciembre, Bogotá.

Diez Canseco, Magdalena

1994 La sabiduría de los orfebres. En: Vich: 183-209, K. Makowski (compilador). Lima, Banco de Crédito del Perú.

Epstein, Stephen

1982 The Prehistoric Copper Smelting Industry at Cerro de los Cementerios, Perú. Analysis of the Product. Masca, The University Museum, University of Pennsylvania, vol.2, NZ̈, pp.58-62. USA.

Epstein, S. e I. Shimada

1983 Metalurgia de Sicán. Una Reconstrucción de la Producción de la 
Aleación de Cobre en El Cerro de los Cementerios, Perú. Beitrage zur Allgemeinen und Vergleichenden Archaologie. Deutschen Archaologischen Instituts, Band 5, Bonn. pp. 379-430.

Foote, H.W.y W. H. Buell

1912 "The composition, structure and hardness of some Peruvian Bronce axes". American Journal of Science. vol. 34, pp. 128-132.

Friedman, A., E. Olsen y J. Bird

1972 "Moche Copper Analyses: Early New World Metal Technology". American

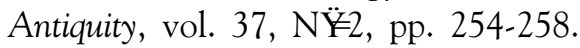
Abril.

Hollister, Víctor

1955 "Procedencia y Composición Química de las Escorias de Chan- Chan". En: Chimor. Boletín del Museo de Arqueología de la Universidad de Trujillo. Año III, vol.I:33-39.

Lechtman, Heather

1971 "Ancient Methods of Gilding Silver: Examples from the Old and the New World".Science and Archaeology. Robert H. Brill, editor. MIT Press, Cambridge, Mass.

1974 "El dorado de metales en el Perú precolombino", Revista del Museo Nacional. Lima: Instituto Nacional de Cultura, Tomo XL, pp. 87-112.

1976 "A Metallurgical Site Survey in the Peruvian Andes". Journal of Field Archaeology, vol.3, N.1:1-42.

1979a "Issues in Andean Metallurgy". En: Pre-Columbian Metallurgy of South America, E.P. Berson Ed. Washington, D.C. Dumbarton Oaks. pp.1-40.

1979b "A pre-Columbian Technique for Electrochemical Replacement Plating of Gold and Silver on Copper Objects". Journal of Metals, vol. 31, NŸ 12, Diciembre, pp. 154-160.

1981 "Copper-Arsenic Bronzes from the
North Coast of Peru". Annals of the New York Academy of Sciences, vol.376:77-122, December 31.

1984a "Metalurgia Superficial Precolombina". Investigación y Ciencia, NŸ95. Prensa Científica, S.A.pp.19-28. Barcelona.

1984b "Andean Value Systems and the Development of Prehistoric Metallurgy". Technology and Culture, vol. 25, Nüt:1-36. Enero.

1988 Traditions and Styles in Central Andean Metalworking. En: The Beginning of the Use of Metals and Alloys. Edited by Robert Maddin. Massachusetts Institute of Technology, pp.344-378.

1991a "La metalurgia precolombina: tecnología y valores", en Los orfebres olvidados de América. Museo Chileno de Arte Precolombino.

1991b "The Production of Copper-arsenic Alloys in the Central Andes: Highland Ores and Costal Smelters?" Journal of Field Archaeology, vol. 18, pp. 43-76.

1996 "El bronce y el Horizonte Medio". 49 Congreso Internacional de Americanistas, Quito, 1997. Boletín Museo del Oro. NŸ41, pp. 3-26. Juliodiciembre,Bogotá.

1997 "El bronce arsenical y el Horizonte Medio", en Rafael Varón y Javier Flores (ed.) Arqueología, Antropología e Historia en los Andes. Homenaje a María Rostworowski.pp. 153-186. Lima.

Lechtman, H.; L. A. Parsons y W. J. Young

1975 Seven matched hollow gold Jaguars from Peru's Early Horizons. Studies in Pre-Columbian Art \& Archaeology, NŸ 6. Dumbarton Oaks. Trustees for Harvard University, Washington, D.C.

Lothrop, Samuel

1953 La metalurgia aborigen de América. Letras. Órgano de la Facultad de Letras de la Universidad Nacional Mayor de San Marcos. NŸ49, p. 83. Lima. 
Mc Ewan, C. y J. Haeberli

2000 "Ancestors Past but Present: Gold Diadems from the Far South COSAT of Peru". en Precolumbian Gold. Technology, Style and Iconography. Ed. C. Mc Ewan. British Museum Press. pp. 16-27.

Makowski, K. y M. I. Velarde

1996 "Taller de Yécala (siglo III/IV): observaciones sobre las características y organización de la producción metalúrgica Vicús”. 49 Congreso Internacional de Americanistas, Quito, 1997. Boletín Museo del Oro. NŸ41, pp. 99-117. juliodiciembre, Bogotá.

Marshall, George

1964 "Examen de Algunas Muestras PreColombinas de Metal". Arqueológicas. NŸ7, Lima.

Mathewson, C.H.

1915 A Metallographic Description of Some Ancient Peruvian Bronzes from Machu Picchu. American Journal of Science, (240): 525-616.

Merkel J., I. Shimada, C. Swann y R. Doonan

1994 "Investigation of Prehistoric Copper Production at Batan Grande, Peru: Interpretation of the Analytical data for ore samples", en D.A. Scott y P. Meyers (ed.). Archaeometrry of Pre-Columbian Sites and Artifacts. pp. 199-227. The Getty Conservation Institute, Marina del Rey, Ca.

Nordenskiold, Erland

1921 "Ancient Colombian Tools of GoldAlloy (Au-Ag-Cu)". Comparative Ethnographical Studies. Vol. 9, Appendix 3a. pp.101-107. Elanders Boktryckeri Aktiebolag, Goteberg,

Petersen, Georg

1970 "Minería y Metalurgia en el Antiguo Perú”. Arqueológicas, NŸ12. Lima.

Ríos, Marcela y Enrique Retamozo

1978 "Objetos de metal procedentes de la Isla de San Lorenzo". Arqueológicas. NŸ17. Lima.

Rovira Llorens, Salvador
1991 "Metales y Aleaciones del Antiguo Perú. Evolución de la Tecnología Metalúrgica”. en Los Incas y el Antiguo Perú. 3000 Años de Historia. Centro Cultural de la Villa de Madrid, FebreroAbril. Tomo I, pp. 82-97. Madrid.

Root, William

1949 "The Metallurgy of the Southern Coast of Perú". American Antiquity. Vol. 15, Nü, pp. 10-37. Salt Lake City.

Rutledge, J.y R. Gordon

1987 "The Work of Metallurgical Artificers at Machu Picchu, Peru". American Antiquity, 52 (3): 578-594.

Schorsch, D.; E. G. Howe y M. T. Wypyski

1996 "Silvered and Gilded Copper Metalwork from Loma Negra: Manufacture and Aesthetics". 49 Congreso Internacional de Americanistas, Quito, 1997. Boletín Museo del Oro. NŸ41, pp. 145-164. Julio-diciembre, Bogotá.

Scott, David

2000 "A Review of Gilding Techniques in Ancient South America", en T. Drayman-Weisser (ed.). Gilded Metals. History, Technology and Conservation. pp. 203-222.

Shimada, Izumi

1985 Perception, Procurement and Management of Resources: Archaeological Perspective. Andean Ecology and Civilization. An Interdisciplinary Perspective on Andean Ecological Complementary. Ed. Masuda, S.; I. Shimada; C. Morris. University of Tokyo Press. Japan. pp. 357-399.

Shimada, I.; A, Gordus; J. A. Griffin y J. F. Merkel 1999 "Sican alloying, working and use of precious metals: an interdisciplinary perspective”. Metals in Antiquity. Ed. By Suzanne Young, Mark Pollard, Paul Budd y Robert Ixer. BAR International Series 792.pp. 301-309.

Shimada, I.; J. A. Griffin y A. Gordus 2000 "The Technology, Iconography and 
Social Significance of Metals: A MultiDimensional Analysis of Middle Sicán Objects", en C. Mc Ewan (ed). Precolumbian Gold. Technology, Style and Iconography. British Museum Press. pp. 28-61.

Shimada, I. y U. Wagner

2001 "Peruvian Black Pottery Production and Metalworking: A Middle Sicán Craft Workshop al Huaca Sialupe". MRS Bulletin, January, pp. 25-30.

Vetter, Luisa

1993 "Análisis de las Puntas de Aleación de Cobre de la Tumba de un Señor de la Elite Sicán, Batán Grande, Lambayeque, Perú". Tesis de Bachiller. Pontificia Universidad Católica del Perú, setiembre.

1996 "El uso del Cobre Arsenical en las Culturas Prehispánicas del Norte del Perú”. 49 Congreso Internacional de Americanistas, Quito, 1997. Boletín Museo del Oro. NŸ41, Julio-Diciembre, pp. 63-81. Bogotá.

2004 "Las Piezas de Metal del Museo de Sitio Arturo Jiménez Borja-Puruchuco". En: Puruchuco y la Sociedad de Lima: un homenaje a Arturo Jiménez Borja. Editores: L. F. Villacorta, L. Vetter y C. Ausejo. (en prensa) "La evolución del Tupu en forma y manufactura desde los Incas hasta el Siglo XIX". 51Congreso Internacional de Americanistas, Santiago de Chile, Chile, julio, 2003.

Vetter, L., P. Carcedo, S. Cutipa y E. Montoya

1997 "Estudio Descriptivo, Metalográfico y Químico de las Puntas de Aleación de Cobre de la Tumba de un Señor de la Elite Sicán, Lambayeque, Perú, empleando Técnicas de Microscopía Optica y Análisis por Activación Neutrónica". Revista Española de Antropología Americana. 27, 23-38. Madrid: Servicio Publicaciones UCM.

Vetter, Luisa y Jesús RUIZ

2003 "Técnicas analíticas en las piezas de metal arqueológicas". 2 Congreso Internacional de Metalurgia de Transformación, Cuzco, 12 al 14 de Nov. CD-Rom.

Vetter, Luisa y Luis Felipe Villacorta

2001 "La Arqueometalurgia de la costa central del Perú: Una perspectiva desde la colección del Museo de Sitio Arturo Jiménez Borja Puruchuco", en Baessler Archiv. NŸ 49. pp. 193-210. Ethnologisches Museum de Berlín. 


\section{ANEXO II}

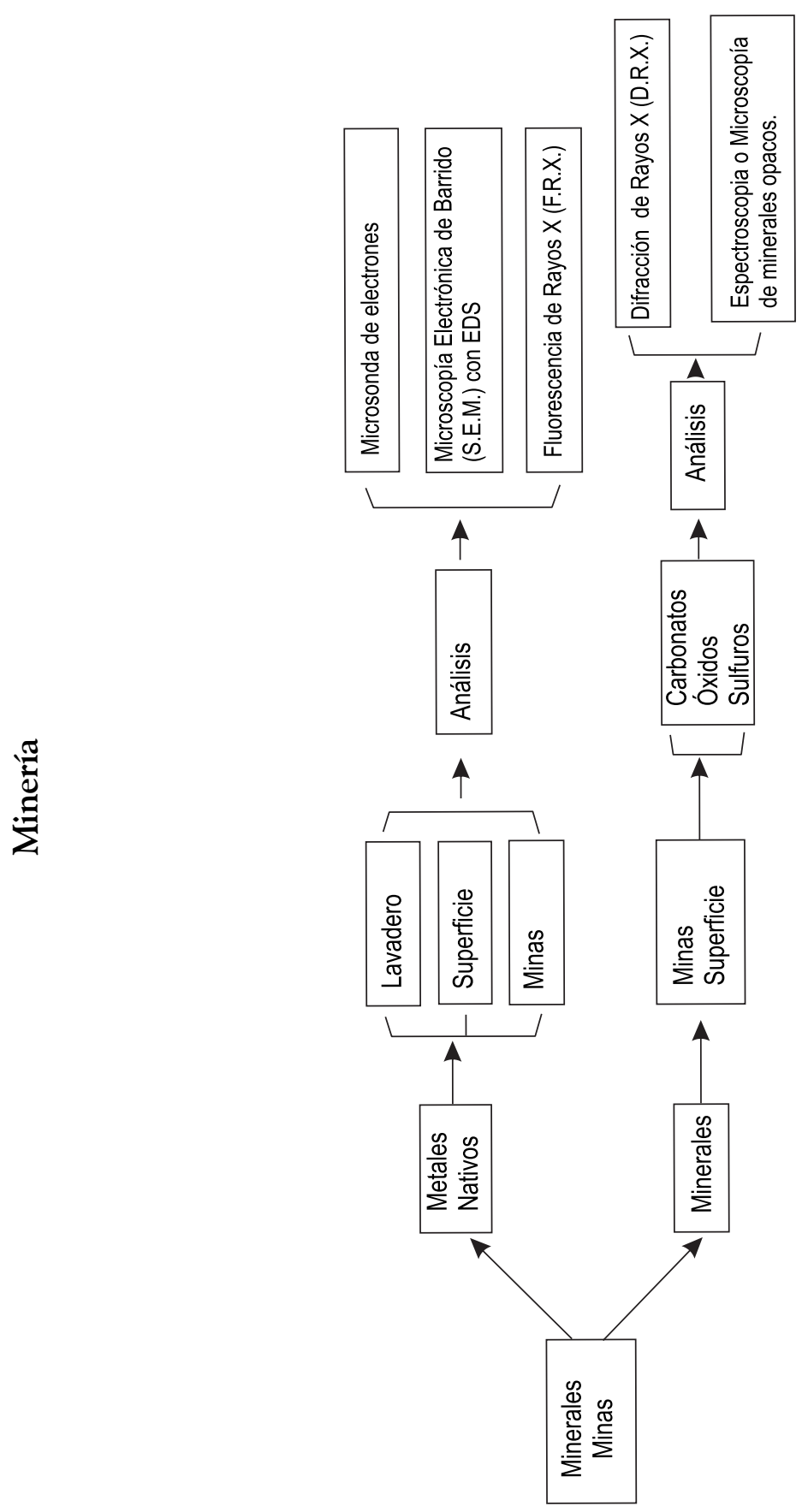




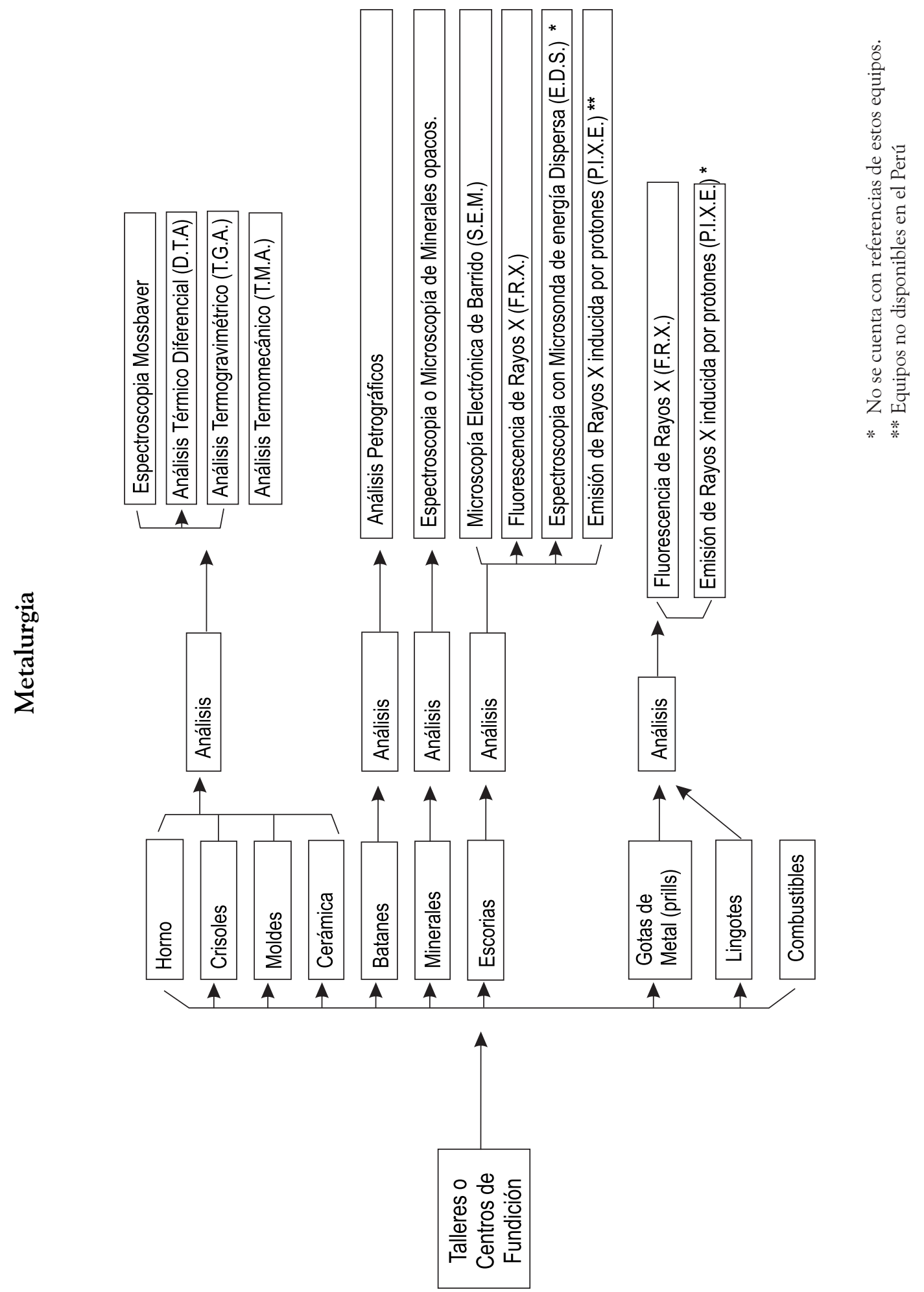




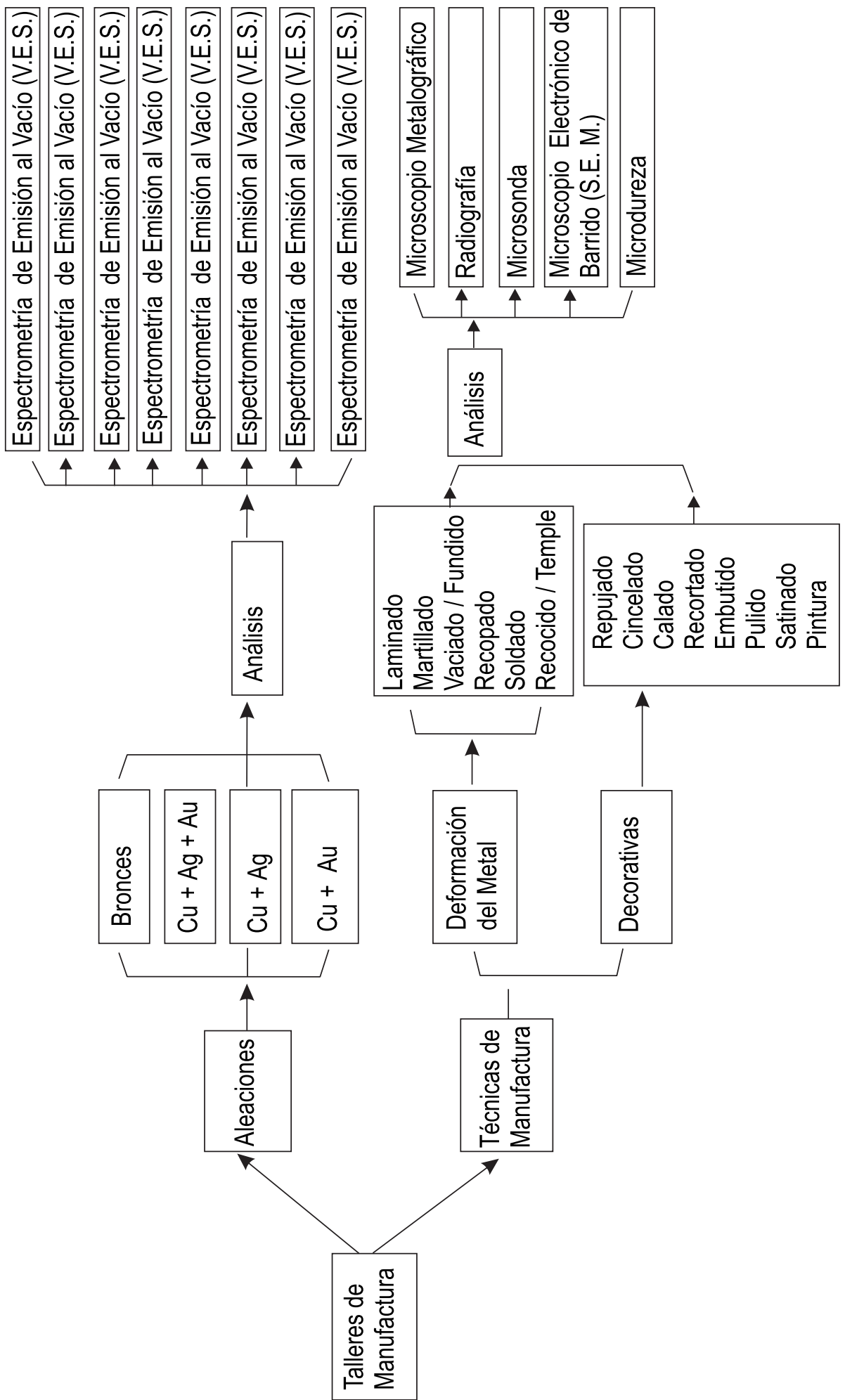

